\section{Utilisation de I'ARN interférence dans le traitement de I'amylose héréditaire due à une mutation de la transthyrétine}

David Adams ${ }^{1}$, Pierre Lozeron ${ }^{2}$, Vincent Algalarrondo ${ }^{3}$
${ }^{1}$ Inserm UMR 788, Université Paris Sud, APHP, centre national de référence des neuropathies amyloïdes familiales (NNERF); CHU Bicêtre, service de neurologie ; 78, rue du général Leclerc 94275 Le Kremlin Bicêtre Cedex, France ;

${ }^{2}$ Inserm UMR 965, Université Paris Diderot, APHP,

CHU Lariboisière, service de physiologie clinique explorations fonctionnelles, 2, rue Ambroise Paré, 75010 Paris, France;

${ }^{3}$ Inserm U769, université Paris Sud, APHP, CHU Antoine Béclère, 157, rue de la porte de Trivaux, 92140 Clamart, France.

david.adams@bct.aphp.fr

pierre.lozeron@lrb.aphp.fr

vincent.algalarrondo@u-psud.fr
> Les neuropathies amyloïdes à transthyrétine (NAH-TTR) sont des maladies héréditaires de l'adulte, de transmission autosomique dominante, liées à une mutation du gène codant pour la TTR. Cette maladie systémique rare, secondaire à des dépôts amylö̈des endoneuraux, est la plus sévère des neuropathies périphériques héréditaires de l'adulte, mortelle en une dizaine d'années. Elle est responsable de polyhandicaps par atteinte progressive des fonctions sensitives, motrices et dysautonomique; elle s'associe à une atteinte cardiaque. Cette affection ubiquitaire concerne également la France [1]. La TTR (ou préalbumine) est synthétisée dans le foie, les plexus choroïdes et l'œil. Elle sert de transporteur à la retinol binding protein (RBP) et à la thyroxine (T4).

Depuis 20 ans, la transplantation hépatique est le seul traitement des NAHTTR qui peut supprimer cette source du variant TTR et stopper la progression de la maladie [2]. Plus de 2000 patients ont été traités dans le monde [3]. Les succès ont été biologique - avec une réduction durable de $98 \%$ de la TTR mutée et donc des dépôts amyloïdes et cliniques, avec l'arrêt de la progression de la neuropathie dans les formes débutantes et l'amélioration de la survie chez les patients jeunes (<50 ans) porteurs du variant Val30Met TTR [4]. L'effet est controversé chez les autres patients [3]. La progression de la neuropathie et de la cardiopathie après transplantation hépatique est en grande partie liée à l'accumulation de la TTR sauvage (WT-TTR) [5, 6] associée à la TTR mutée d'après l'analyse des dépôts amyloïdes par spectrométrie de masse; cette accumulation se majore après 50 ans et ce malgré la transplantation hépatique [7].

Le besoin de médicaments pour les NAHTTR se justifie car la transplantation hépatique: (1) ne concerne que 2/3 des patients atteints de NAH-TTR en France (ceux dont l'âge est < 70 ans) [1]; (2) ne modifie pas la survie des patients qui ne sont pas porteurs d'une mutation de type Val30Met; (3) n'est possible qu'après une période d'attente d'un greffon de 6 à 12 mois pendant laquelle la maladie continue à progresser. Les stabilisateurs cinétiques de la TTR agissent en se fixant sur le site de la thyroxine, stabilisant le tetramère et évitant sa dislocation en dimères ou monomères amyloïdogènes. Une étude multicentrique a ainsi montré l'efficacité du tafamidis pour ralentir la progression des formes toutes débutantes de Val30Met de NAH-TTR, lui permettant d'obtenir l'autorisation de mise sur le marché en Europe [8].

\section{Principe de l'ARN interférence}

L'interférence d'ARN ( $A R N i$ ) est un mécanisme cellulaire endogène qui contrôle l'expression de gènes; il fait intervenir des petits ARN qui se lient au complexe multiprotéique induit par
I'ARN (RISC, RNA-induced silencing complex) et aboutissent, en le clivant, à l'inactivation de I'ARN messager cible (ARNm), empêchant sa traduction en protéine [9]. Dans le cas de la TTR, I'ARNi cible une séquence conservée dans la région 3' non transcrite de l'ARNm, affectant les deux formes, mutée et non mutée, de la protéine. Grâce à des formulations de nanoparticules lipidiques, la biodistribution de ces ARNi après administration intraveineuse (iv) a surtout lieu dans le foie (hépatocytes) (Figure 1); le système nerveux central et l'œil sont épargnés. Un petit ARNi anti-TTR puissant a été identifié, qui a été encapsulé dans deux formulations distinctes de nanoparticules de lipides de $1^{\mathrm{e}}$ et $2^{\mathrm{e}}$ générations, produisant respectivement ALN-TTROI et ALN-TTRO2 (ALN pour Alnylam Pharmaceuticals) [10].

Des études chez des primates non humains et deux essais cliniques de phase 1 ont été réalisés pour déterminer l'activité et les puissances relatives de ces deux formulations dans la baisse des taux de TTR. Les patients reçoivent une prémédication la veille et le jour du traitement pour réduire le risque de réactions liées à la perfusion, et les formulations, ALN-TTRO1 ou ALN-TTRO2, sont administrées par voie iv pendant 15 et 60 minutes respectivement [10].

Les patients éligibles étaient âgés de plus de 18 ans, et porteurs d'une neuropathie de gravité mineure à modérée ; 


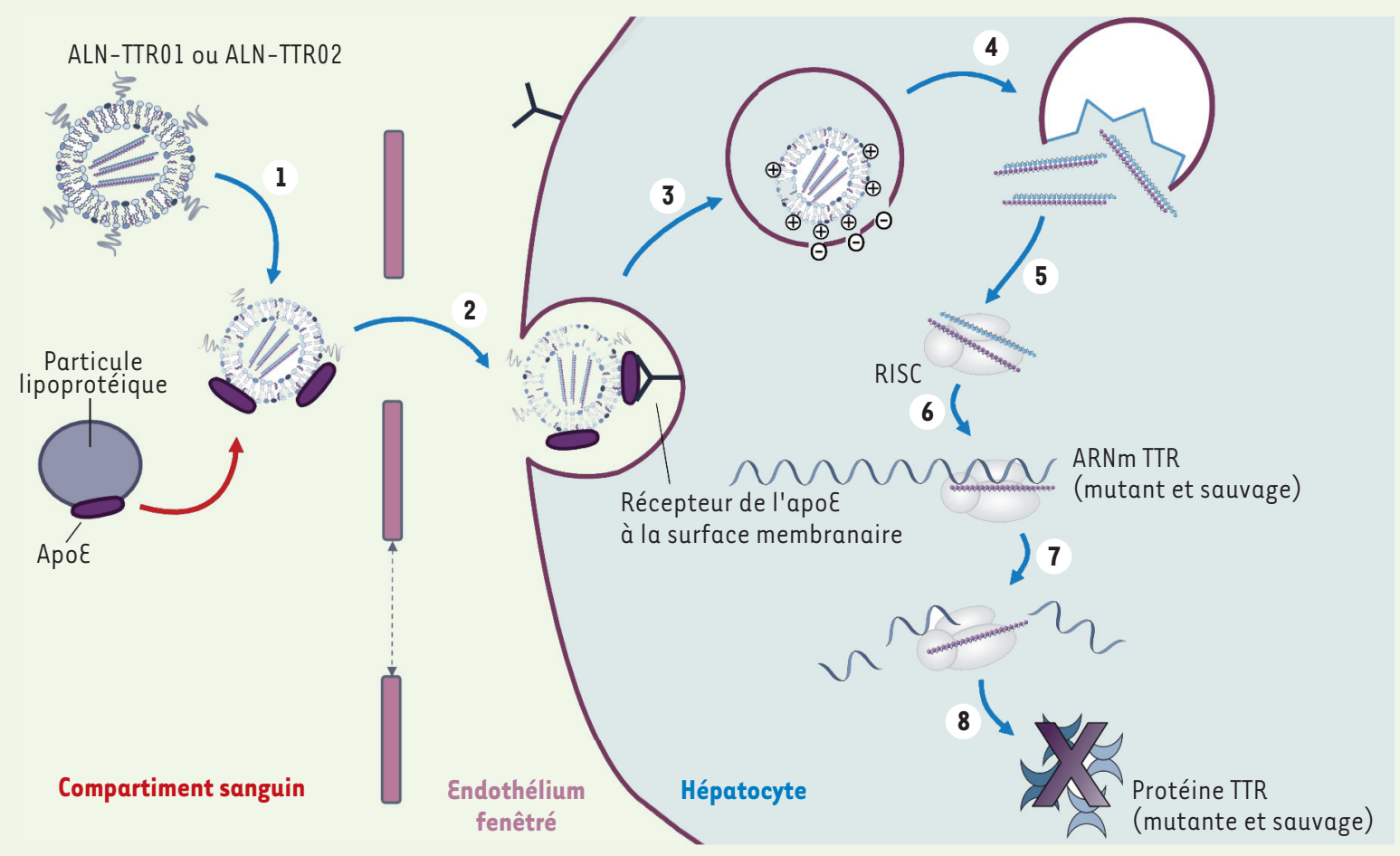

Figure 1. Mécanisme de livraison des ARNi aux hépatocytes et suppression de la protéine TTR médiée par les ARNi. ALN-TTR01 et ALN-TTR02 sont des formulations de nanoparticules lipidiques (NPL) de première et seconde génération du même ARNi ciblant l'ARN messager (ARNm) des TTR mutée et sauvage. Après administration intraveineuse, les NPL sont opsonisées par l'apoliprotéine $\varepsilon$ (apoE) (1), puis entrent dans le foie à partir de la circulation grâce aux vaisseaux fenêtrés où elles se lient aux récepteurs de l'apoz à la surface des hépatocytes (2). Les NPL sont alors intégrées par endocytose et le $\mathrm{pH}$ acide de la vésicule endocytaire aboutit à une protonation du composant lipidique ionisable du NPL (3). La NPL positivement chargée fusionne alors avec la membrane endosomale négativement chargée, aboutissant à la sortie de l'ARNi dans le cytoplasme (4) et son chargement sur le complexe inducteur du silence d'ARN (RISC) (5). Les deux brins d'ARNi sont alors séparés, et le brin antisens complexé au RISC se lie à la même séquence dans la région non transcrite des ARNm de la TTR mutée et sauvage (6). L'endonucléase Argonaute 2 (Ago2) au sein du RISC induit le clivage de l'ARNm (7), induisant l'inactivation des ARNm des TTR mutée et sauvage et la suppression de la protéine TTR (8) (adapté de [10]) (copyright (c) [2013] Massachusetts Medical Society. Reprinted with permission).

le diagnostic d'amylose à TTR était prouvé par biopsie, le score de Karnofsky était $\geq 60^{\mathrm{l}}$, l'indice de masse corporelle entre 18,5 et 33 , une classe de dyspnée $\mathrm{NYHA} \leq \mathrm{a} \mathrm{II}^{2}$, et des fonctions hépatique, rénale et thyroïdienne normales.

\footnotetext{
1 II s'agit d'une échelle qui évalue la dépendance des patients. Par exemple, un score de 100 n'indique aucune évidence de maladie; score de 80 , le patient est capable d'exécuter une activité normale mais avec effort ; quelques signes apparents de la maladie; un score de 60 reflète une dépendance occasionnelle, mais le patient est capable d'assurer la plupart de ses propres besoins.

2 II s'agit de la classification de la New York Heart Association (NYHA) de l'insuffisance cardiaque. Le stade I ne montre pas de limitation; le stade II une petite limitation des capacités physiques: le patient n'a pas de symptômes au repos, mais des efforts normaux provoquent fatigue, palpitations ou dyspnée.
}

\section{Efficacité des ARNi}

Une baisse du taux de TTR, rapide, dosedépendante et durable a été observée dans les deux essais. Dans l'étude TTROl, chez les quatre patients avec la mutation Val30Met recevant une dose de $1 \mathrm{mg} / \mathrm{kg}$, les 2 formes mutée et non mutée de la TTR sont réduites dans les mêmes proportions, avec une cinétique de diminution et de rétablissement identique. Le patient avec la mutation Ser77Phe a une réponse robuste; le taux de TTR chute de plus de $50 \%$ dès $J 2$ et jusqu'à $81 \%$ à $J 10$. Une réduction de $50 \%$ persistait à $\mathrm{J} 28$, le retour au taux de base n'étant atteint qu'à $J 70$.

Pour la formulation ALN-TTR02, une réduction de TTR a été observée chez tous les participants recevant les doses de 0,15 à 0,5 mg/kg. Celle-ci était rapide, puissante et durable, significative par rapport au placebo $(P<0,001)$ et persistait jusqu'à J28. La réduction maximale du taux de TTR pour les participants recevant $0,15 \mathrm{mg}, 0,3 \mathrm{mg}$ et $0,5 \mathrm{mg}$ par kg était respectivement de $85,7 \%, 87,6 \%$ et $93,8 \%$. Celle-ci se maintenait à $\mathrm{J} 28$ avec une baisse du taux de TTR de $56,6 \%$ et $67,1 \%$ pour des participants recevant $0,15 \mathrm{mg}$ et $0,3 \mathrm{mg} / \mathrm{kg}$. Ces réductions de TTR par ALN-TTROl et ALN-TTRO2 étaient corrélées aux changements des taux sériques de RBP (retinol binding protein) et de vitamine $A$ [10]. 


\section{Un mode d'action spécifique de ALN-TTRO2}

Pour démontrer l'effet spécifique d'ALNTTR02 - indépendamment de la formulation de nanoparticules lipidiques -, la TTR a été dosée dans un essai de phase 1 chez un groupe de volontaires sains ayant reçu une dose unique de $0,4 \mathrm{mg} / \mathrm{kg}$ d'ALN-PC, ou d'ALN-PC ARNi contrôle, dans lequel I'ARNi ciblait PCSK929 (une cible pour réduire le taux de cholestérol) avec la même formulation de nanoparticules lipidiques que I'ALN-TTRO2; aucun effet sur le taux de TTR n'a été observé. Une autre preuve a été apportée par le test 5' RACE (amplification rapide de terminaisons d'ADN complémentaires) effectué sur des prélèvements sanguins obtenus chez des participants de l'essai recevant une dose de $0,3 \mathrm{mg} / \mathrm{kg}$ d'ALNTTR02. Alors que I'ARNm de TTR a été détecté dans les échantillons avant l'administration d'ALN-TTRO2 et $24 \mathrm{~h}$ après, le produit de clivage de l'ARNm de TTR dans la circulation extracellulaire ne l'était que dans des échantillons après administration d'ALN-TTRO2 [10].

\section{La sécurité des ARNi}

L'utilisation d'ALN-TTRO1 et ALN-TTRO2 n'a induit aucun effet indésirable sérieux lié à la drogue et l'essai n'a été arrêté chez aucun patient [10]. Aucune perturbation hématologique, hépatique, ou des fonctions rénale ou thyroïdienne n'a été notée. Des réactions légères à modérées sont survenues chez 20,8\% des participants recevant des doses d'ALN-TTROl supérieures à $0,4 \mathrm{mg} / \mathrm{kg}$. Une réaction modérée liée à la perfusion est survenue chez le patient qui a reçu $A L N-T T R 02$ à une dose de $0,5 \mathrm{mg} / \mathrm{kg}$ (incidence globale 7,7\%). Les réactions légères se sont résolues spontanément; les réactions modérées ont entrainé l'arrêt temporaire de la perfusion avec ou sans administration de glucocorticoïdes, permettant la reprise de l'administration de la drogue à un débit plus lent. Les réactions observées lors de la perfusion dans les deux essais n'ont été associées à aucune induction de cytokines proinflammatoires. Aucun anticorps dirigé contre le composant lipidique pégylé d'ALN-TTR02 n'a été détecté chez les participants à l'essai.

En conclusion, ce travail montre que I'utilisation d'ARN interférents ouvre une nouvelle ère thérapeutique pour l'amylose à transthyrétine. La capacité d'ALN-TTRO2 à abaisser les taux des protéines mutées et non mutées offre un avantage indiscutable sur la transplantation hépatique, qui n'affecte que la production de protéines mutées. Une étude randomisée de phase 3 , en double aveugle contrôlée contre placebo, multinationale, a débuté (référence sur Clinicaltrial.Gov identifiant: NCT01960348). Les injections d'ALNTTR02 sont faites toutes les 3 semaines pendant 18 mois. L'essai évalue l'efficacité clinique et la sécurité de la réduction à long terme des TTR chez des patients atteints de NAH-TTR. Le critère principal de mesure est la différence dans le changement du score composite de base de neuropathie modifiée $(\mathrm{mNIS}+7)$ à 18 mois d'intervalle entre les groupes recevant ALN TTR02 ou le placebo. Le centre de référence national des neuropathies amyloïdes familiales (NNERF) coordonnera pour la France le déroulement de l'étude. $\diamond$
Use of RNAi in the treatment of hereditary amyloidosis due to mutation of transthyretin

\section{LIENS D'INTÉRÊT}

D. Adams est consultant pour ALNYLAM et ISIS et a reçu des compensations financières pour des symposiums avec l'entreprise Pfizer. P. Lozeron a reçu une compensation financière personnelle de l'entreprise FOLD-RX pour sa participation à l'étude FX-005. V. Algalarrondo déclare participer à des interventions ponctuelles pour l'entreprise Medtronic.

\section{RÉFÉRENCES}

1. Adams D, Lozeron P, Theaudin M, et al. Regional difference and similarity of familial amyloidosis with polyneuropathy in France. Amyloid 2012 ; 19 Suppl 1 : 61-4.

2. Adams D, Samuel D, Goulon-Goeau C, et al. The course and prognostic factors of familial amyloid polyneuropathy after liver transplantation. Brain 2000 ; 123 ( Pt 7) : 1495-504.

3. Wilczek HE, Larsson M, Ericzon BG, Fapwtr. Long-term data from the Familial Amyloidotic Polyneuropathy World Transplant Registry (FAPWTR). Amyloid 2011 ; 18 Suppl 1 : 193-5.

4. Yamashita T, Ando Y, Okamoto S, et al. Long-term survival after liver transplantation in patients with familial amyloid polyneuropathy. Neurology 2012 ; $78: 637-43$.

5. Liepnieks JJ, Zhang LQ, Benson MD. Progression of transthyretin amyloid neuropathy after liver transplantation. Neurology $2010 ; 75$ : 324-7.

6. Yazaki M, Mitsuhashi S, Tokuda T, et al. Progressive wild-type transthyretin deposition after liver transplantation preferentially occurs onto myocardium in FAP patients. Am J Transplant 2007 ; $7: 235-42$.

7. Ihse $\varepsilon$, Suhr $O B$, Hellman U, Westermark P. Variation in amount of wild-type transthyretin in different fibril and tissue types in ATTR amyloidosis. J Mol Med 2011 $89: 171-80$.

8. Coelho T, Maia LF, Martins da Silva A, et al. Tafamidis for transthyretin familial amyloid polyneuropathy a randomized, controlled trial. Neurology 2012; 79 : 785-92.

9. Elbashir SM, Harborth J, Lendeckel W, et al. Duplexes of 21-nucleotide RNAs mediate RNA interference in cultured mammalian cells. Nature 2001 ; 411 : 494-8.

10. Coelho T, Adams D, Silva A, et al. Safety and efficacy of RNAi therapy for transthyretin amyloidosis. New Engl J Med 2013 ; 369 : 819-29.

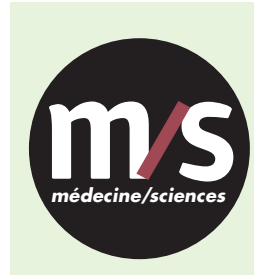

Tarifs d'abonnement $m / s-2014$

Abonnez-vous

à médecine/sciences
$>$ Grâce à $m / s$, vivez en direct les progrès des sciences biologiques et médicales

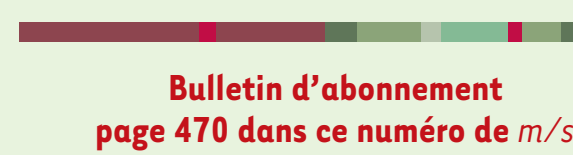

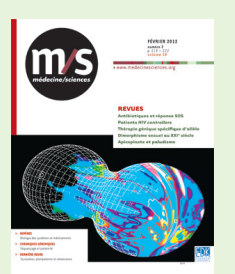

